\title{
Implementation of a Scene Expansion Mechanism Using an Event Sequence: As a Mechanism in an Automatic Narrative Generation Game
}

\author{
Jumpei Ono and Takashi Ogata
}

\begin{abstract}
The goal of this study is to develop a game that generates a story automatically based on the story creation process used in Table-top role playing games (TRPGs). TRPG is an analog game that employs the creativity of the game player. We have already produced a mechanism for developing a scene sequence from a mutual communication between game master (GM) and player (PL) experimentally. This mechanism generates a story constructed from a few scenes. Scenes are parts of a story that are separated by a temporal and spatial border. In this paper, we introduce a mechanism to expand scenes by using event sequence information with the goal of generating a long story. The event sequence information is obtained using a semi-automatic event sequence acquisition system that was developed in previous work.
\end{abstract}

Index Terms-Automatic narrative generation game, event sequence, script, table-top role playing game.

\section{INTRODUCTION}

The goal of this study is to develop a game that generates a story automatically based on a process involving the communication between a "Game Master (GM)" and "Player (PL)" in a Table-top Role Playing Game (TRPG) [1]. The TRPG, invented by Gary Gygax [2], is an analog game that employs the creativity of the game players. For example, there is a study that examined "creativity" from six viewpoints, i.e. story, acting, gaming, problem-solving, game-world [3], and stimulated creativity of the game user by processing the communication between GM and PL in the game system, which the authors suggest consciously aims at generating many kinds of stories.

As part of the trial run of the automatic story generation game, the mechanism to generate a scene sequence from the communication between GM and PL was developed [4]. This mechanism generates the story, which is constructed by a few scenes (scenes are parts of a story that are separated by a temporal and spatial border). A mechanism that expands scenes by applying the knowledge of an event sequence to the mechanism has been introduced in this paper. The purpose is to generate a longer narrative. The event sequence information is created using a semi-automatic event sequence

Manuscript received September 15, 2016; revised December 23, 2016. The authors are with Iwate Prefectural University, Sugo, Takizawa, Iwate, 152-52, Japan (e-mail: g236m001@ s.iwate-pu.ac.jp, t-ogata@iwate-pu.ac.jp). acquisition system that was developed in previous work [5].

\section{BACKGROUND}

In this paper, a new mechanism that combines two experimentally developed mechanisms has been suggested. This section outlines both these mechanisms and the system that is created using these mechanisms.

\section{A. What Game Is in the Paper}

Digital games based on TRPG are announced in various mediums so far. These are the games that brought how to play in TRPG close to conventional digital games. The PL is the user and the GM is a computer in those games. The game proposed here is intended for the game system, which we modeled closely after the structure of a TRPG. The game user assumes a role unlike the GM and PL. In an extreme example, we could imagine a game in which the user has almost no participation.

In the study that examined the story creation process of a TRPG, a model [6] for devotional storytelling is suggested for use by story participants who take on the roles of one of the characters. An interviewer shares story space with a narrator and demonstrates controlling the progress of the story interactively in a TRPG. There is a study that examined the power a game participant has to shape the story in a TRPG across three varieties of games in the TRPG genre. The study includes an example [3] that examined "creativity".

\section{B. A TRPG-Based Automatic Narrative Generation Game}

The automatic narrative generation game generates a story by fleshing out a rough framework of it using various methods. We call a generated story a "scenario" here. The scenario consists of a "world setting" and a "scene sequence". The former is the set of the information necessary to flesh out the story, and it includes characters, objects, locations and times, and the set of limitations and rules to be applied to those elements. The scene sequence is a set of multiple scenes. The rough framework of the story mentioned above is a scene sequence, and the scenario that we finally flesh out is a scene sequence. The individual scenes are constructed as an action sequence of the characters in a range that is divided temporally and spatially in a scenario. Table 1 has a summary of terms related to TRPG.

Fig. 1 shows the generation mechanism; the overall processing is divided into three phases: (Step.1) preparations, (Step.2) story generation, and (Step.3) result output. The authors developed the mechanism for Step.2 via their experiments. 
TABLE I: TERMS IN THE NARRATIVE GENERATION GAME

\begin{tabular}{|l|l|c|}
\hline \multicolumn{1}{|c|}{ Terms } & \multicolumn{1}{|c|}{ Role } & $\begin{array}{c}\text { Terms in } \\
\text { TRPG }\end{array}$ \\
\hline $\begin{array}{l}\text { Game master } \\
(\mathrm{GM})\end{array}$ & $\begin{array}{l}\text { To control the preparations for and the } \\
\text { progression of the game. Usually alone. }\end{array}$ & GM \\
\hline Player (PL) & $\begin{array}{l}\text { To play characters in the game. There are } \\
\text { multiple PLs. }\end{array}$ & PL \\
\hline Scenario & $\begin{array}{l}\text { Overall information that connects world } \\
\text { setting and scene sequence. }\end{array}$ & - \\
\hline World setting & $\begin{array}{l}\text { Information that constitutes the setting of } \\
\text { the game and limitation. } \\
\text { To include characters, things, locations, } \\
\text { time and restrictions in the game. }\end{array}$ & System \\
\hline $\begin{array}{l}\text { Scene } \\
\text { sequence }\end{array}$ & $\begin{array}{l}\text { Sequence of several scenes. This sequence } \\
\text { includes the condition of the change in two } \\
\text { scenes. }\end{array}$ & Scenario \\
\hline Scene & A spatial and temporal section in a story. & Scene \\
\hline
\end{tabular}

By using this trial mechanism, a scene sequence is expanded when GM shows a scene sequence and PL suggests a scene. Fig. 2 illustrates the process.

However, this trial mechanism does expand a scene into plural events. Therefore, in this article, the scene development mechanism by the event sequence that uses the mechanism to speak with the next section experimentally has been used.

A scene is a spatial and temporal section in a story. Furthermore, the scene is constructed by one or more events. An event points at movement and the change with any component in the story.

\section{Integrated Narrative Generation System}

The Integrated narrative generation system (INGS) [7] being developed is a system that integrates the mechanism of story generation that was systematically developed by us, and it is the basis of other application systems. The automatic narrative generation game uses various mechanisms of the INGS.

The INGS aims at storing pieces of the story (the special pieces of the story are the whole structure of the story) in various particle sizes, by classifying and arranging those pieces systematically, without limiting the pieces of a story and a discourse to a particular type and genre beforehand. A knowledge base is called a story contents knowledge base for its purpose. Fig. 3 is an example of the knowledge. The displayed knowledge is a constructed element that includes conceptual dictionaries. The conceptual dictionaries are dictionaries that include noun concepts or verb concepts structurally.

\section{Semi-automatic Event Sequence Acquisition System}

The automatic acquisition of every possible piece of the story contents knowledge base was one of the purposes, and the trial manufacture of a semi-automatic acquisition tool of the event sequences was the primary objective [5]. An event sequence structures events systematically. The acquisition tool mentioned above acquires script knowledge in story content knowledge bases [7].

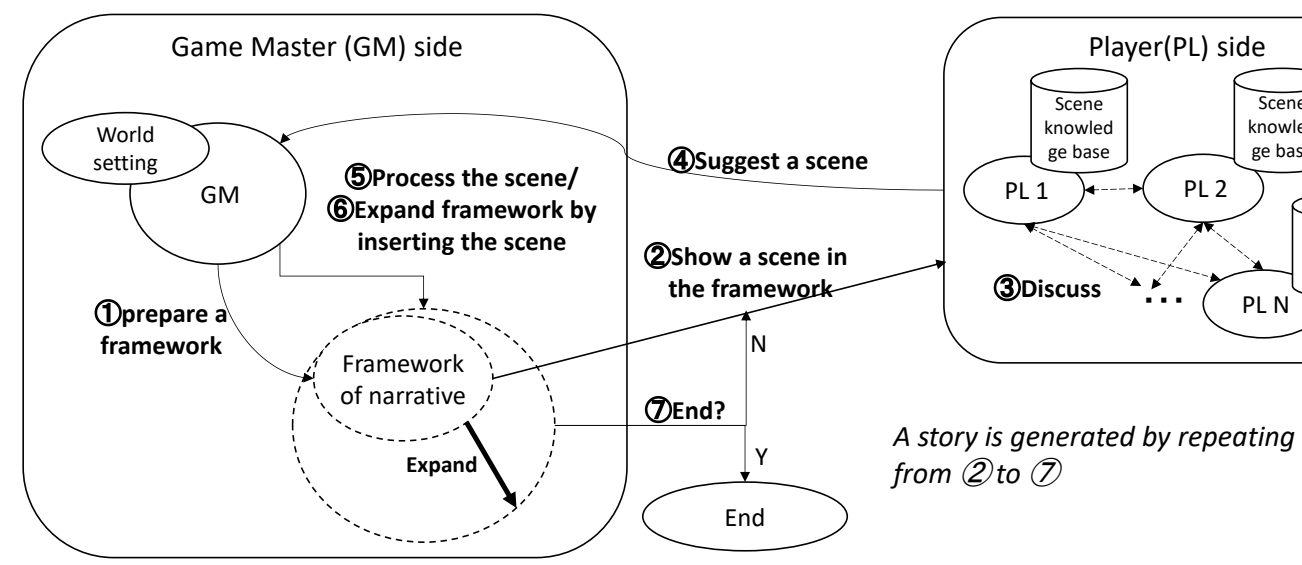

Fig. 1. A story generation model in the Table-top role playing game.

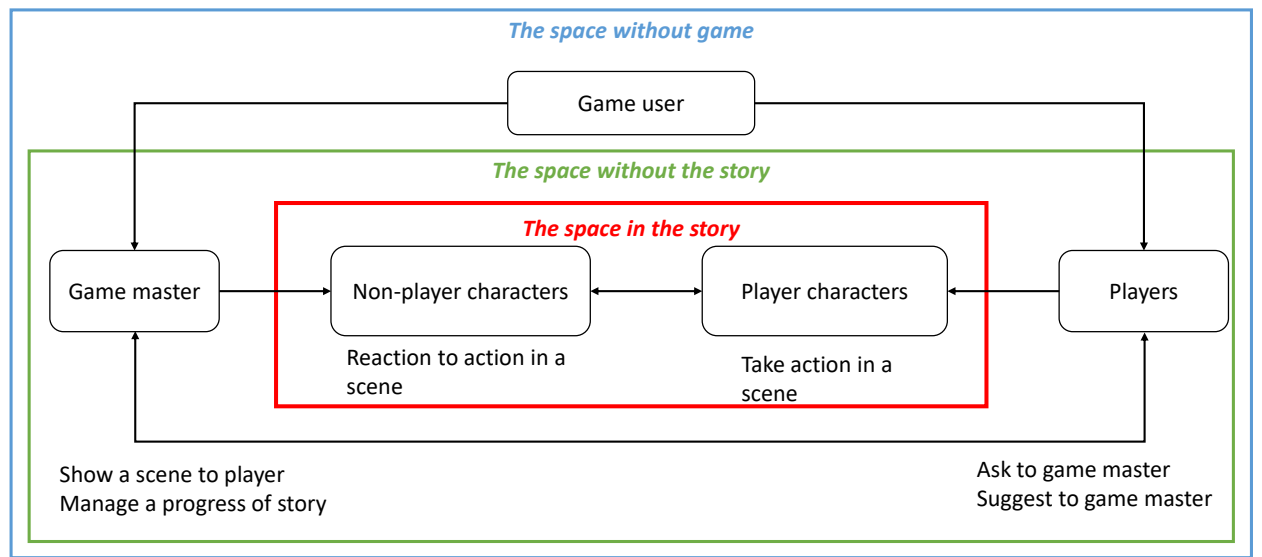

Fig. 2. The overview of an interaction between game master and player.

In the semi-automatic acquisition of script knowledge [5], the user selects a verb concept from verb concepts displayed by the tool. The user writes a script that includes two or more events to expand it concretely in natural language. The tool 


\section{B. Result}

Using the input that was used by the trial manufacture of the scene sequence expansion mechanism [4], a story was generated using the scene expansion mechanism.

First, a user builds event sequences that link the verb concept (scene) included in a scene sequence input to scene sequence expansion mechanism and the verb concept (scene) included in the knowledge base that PL has. Next, with the expansion of the scene sequence, the mechanism develops the scene using the event sequence that the user built.

\section{1) Constructing of event sequence knowledge}

Fig. 6 shows the generation process of the event sequence, and Fig. 7 is a completed event sequence. Six event sequences ware made from verb concepts ("kidnap", "dispute", "be-left-intact", "examine", "shoot", "fight"), which are included in the scene sequence later and the knowledge base that PL has.

\section{2) Expanding a scene using event sequence knowledge}

Fig. 8 is input, and Fig. 9 is the result of the expansion of the scene sequence and the expansion of scenes. By this result, the knowledge that has been already stored by a story contents knowledge base is used as well as the event sequence that a user built ("fight" that was inserted by suggestion of PL was expanded by using the knowledge that has already been stored in this result).

「誘拐する 1」を, 二つ以上の文から成る事象連鎖に展開してく ださい。[Please, you change "kidnap" to event sequence that conform two more sentences.]

なお、「誘拐する 1」とは「男が子供を誘拐する」という意味で す。[And, "kidnap" means "A man kidnaps a child".]

入力をどうぞ。[Please, you input sentences.]

>>男が子供を追う。男が子供を捕らえる。男が逃げる。[A man chases a child. The man catches the child. The man escape.] Fig. 6. Example of scene expansion.

\begin{tabular}{|c|c|}
\hline \multicolumn{2}{|c|}{ 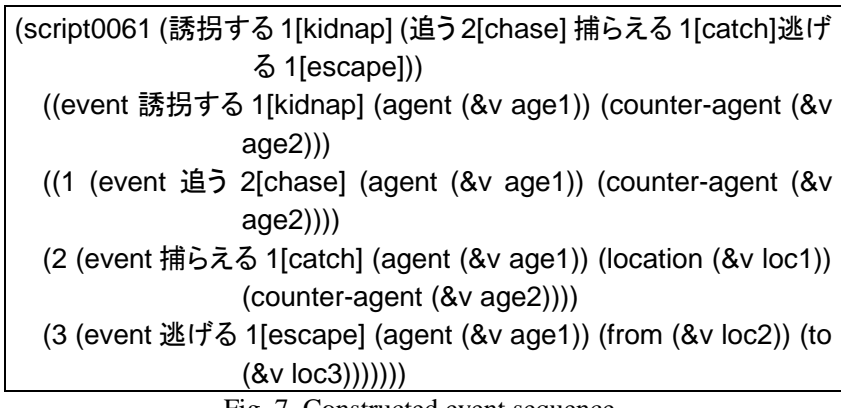 } \\
\hline \multicolumn{2}{|r|}{ Fig. 7. Constructed event sequence. } \\
\hline $\begin{array}{l}\text { ene } \\
\text { quenc }\end{array}$ & 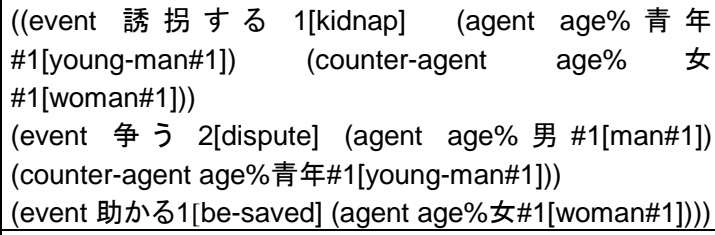 \\
\hline & $\begin{array}{l}\text { ) (instance-of 青年@男 } \\
\text { 持[possession] nil) (健康 } \\
\text { 載 業 [job] 警 官 @ 警 官 }\end{array}$ \\
\hline
\end{tabular}

Fig. 8. Input data for scene sequence expansion mechanism.

In this paper, we suggest the system which performs story generation by burying the blank of the framework of story. This mechanism is one of two funs that automatic narrative generation game which the authors aims for has. Another fun is to bury a blank in form to betray the ending included in the frame daringly. As a result, the story that GM does not expect is completed. The mechanism that we spoke here is mechanism to become the premise.

\section{((誘拐する 1[kidnap]}

(event 追う2[chase] (agent age\%青年\#1[young-man\#1]) (counter-agent age\%女\#1))

(event 捕らえる 1[catch] (agent age\%青年\#1[young-man\#1]) (location loc\%通り\#1[street\#1]) (counter-agent age\%女\#1))

(event 逃げる 1[escape] (agent age\%青年\#1[young-man\#1]) (from loc\%裏道\#1[back-street\#1]) (to loc\%家\#1[house\#1])))

(調査する 1 [examine]

(event 調べる[check] (agent age\%男\#1[[man\#1]]) (counter-agent age\%女\#1[woman\#1]))

(event 発見する2[find] (agent age\%男\#1[man\#1]) (counter-agent age\%女\#1[woman\#1])))

(争う2[dispute]

(event 殴る 2[strike] (agent age\%青年\#1[young-man\#1]) (counter-agent age\%男\#1[man\#1]))

(event 避ける 1[avoid] (agent age\%男\#1[man\#1]) (counter-agent age\%青年\#1[young-man\#1]))

(event 殴る 2[strike] (agent age\%男\#1[man\#1]) (counter-agent age\%青年\#1[young-man\#1])))

(戦う 3[fight]

(event 襲う 2[attack] (agent age\%青年\#1[young-man\#1]) (counter-agent age\%男\#1[man\#1]))

(event 避ける 1[avoid] (agent age\%男\#1[man\#1]) (counter-agent age\%青年\#1[young-man\#1]))

(event 引き抜く2[entice] (agent age\%男\#1[man\#1]) (object obj\%剣\#1[sword\#1]) (from obj\%鞘\#1[sheath\#1]))

(event 突く2[attack] (agent age\%男\#1[man\#1]) (counter-agent age\%青年\#1[young-man\#1]))

(event 避ける 1[avoid] (agent age\%青年\#1[young-man\#1]) (counter-agent age\%男\#1[man\#1]))

(event 反撃する 1[counterattack] (agent age\%青年\#1[young-man\#1]) (counter-agent age\%男\#1[man\#1])))

(助かる 1 [be saved]

(event 帰る 2[come-home] (agent age\%女\#1[woman\#1]) (from loc\%家\#1[house\#1]))

(event 喜ぶ1[be-glad] (agent age\%女\#1[woman\#1]))))

Fig. 9. Generated scene sequence.

IV. CONCLUSION

In this paper, a scene development mechanism using event sequences was proposed. This is a mechanism for automatic 
narrative generation that includes a generative model to represent the communication between GM and PL in a Table-top role playing game. This proposed mechanism is a part of the mechanism that has been proposed [4].

The above mechanism presents a scene as part of a scene sequence, and the mechanism outlined in this paper is comprised of the user making an event sequence and going through the scene development process.

The coherence examination between events or scenes in a story generated by questionnaire survey is planned for the future.

Furthermore, we consider acquiring knowledge from a generated story. The structure that is decomposed into the structure of the story itself or a certain size is integrated into a knowledge base, which is a trial to bring about new knowledge for structure generation of the stories by performing the resolution of a formed story or the combination of two stories [8].

\section{REFERENCES}

[1] J. Ono and T. Ogata, "Architecture of a narrative generation system based on a TRPG model: The use of an integrated narrative generation system for knowledge acquisition (preliminary version)," Bulletin of Networking, Computing, Systems, and Software, vol. 5, no. 1, pp. 40-48, 2016.

[2] M. Witwer, "Empire of imagination: Gary Gygax and the birth of dungeons \& dragons, bloomsbury," 2016.

[3] K. Bergström, "Creativity rules. How rules impact player creativity in three tabletop role-playing games," International Journal of Role-Playing, vol. 3, pp. 4-17, 2012.

[4] J. Ono and T. Ogata, "A prototype system of a scene sequence expansion mechanism in an automatic narrative generation game based on TRPG method," in Proc. the 30th Annual Conference Japanese society for Artificial Intelligence, 2016.

[5] T. Arai, J. Ono, and T. Ogata, "Semi-automatic generation of event sequence knowledge for narrative generation: The use in an integrated narrative generation system," in Proc. the 30th Annual Conference Japanese Society for Artificial Intelligence, 2016.

[6] Hoshino and J. Storytelling, Journal of Japanese Society for Artificial Intelligence, vol. 19, no. 1, pp. 29-34, 2004.

[7] T. Ogata, "Computational and cognitive approaches to narratology from the perspective of narrative generation," IGI Global, pp. 1-73, 2016.

[8] J. Ono and T. Ogata, "Automatic acquisition from the web of expression elements in a narrative generation system," in Proc. The 27th Annual Conference of the Japanese Society for Artificial Intelligence, 2013.

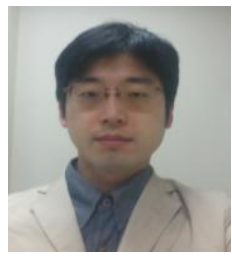

Jumepi Ono received his bachelor of information science in Iwate Prefectural University in 2010. He received his M.S. in Iwate Prefectural University in 2014. He is currently pursuing his Ph.D. from the Graduate School of Iwate Prefectural University. His research interests are narrative generation and games.

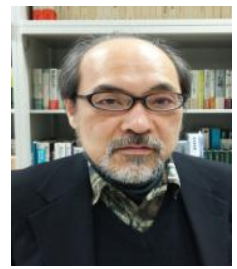

Tkashi Ogata received his bachelor of social science in Waseda University in 1983. He received his M.S. in Tsukuba University in 1992 and his Ph.D. in the University of Tokyo in 1995. He has industrial experience since 1983 at software development companies. Having experienced associate professor of the Faculty of Engineering at Yamanashi University since 1997, he is Professor of the Faculty of Software and Information Science at Iwate Prefectural University since 2005. His major research interests include artificial intelligence, cognitive science, natural language processing, narratology and literary theories, an interdisciplinary approach to the development of narrative generation systems based on AI and narratology, and the application to narrative creation and business. He is a member of the Japanese Society for Artificial Intelligence, the Japanese Cognitive Science Society (committee), and the Japanese Association for Natural Language Processing. He is also a main manager of Literature, Cognition and Computer research group at the JCSS. He received JSAI best paper award (1996), best paper award of Japan Academy of Advertising (1996), and other academic awards. 